\title{
Toxicological Analysis of Warfarin in an Adult Developing Diffuse Alveolar Hemorrhage: A Case Report and Literature Review
}

\author{
Ismail Ethem Goren (Corresponding author) \\ Department of Forensic Medicine, Faculty of Medicine, Cukurova University \\ PO box 01330, Adana, Turkey \\ E-mail: ismailethemgoren@gmail.com \\ Nebile Daglioglu \\ Department of Forensic Medicine, Faculty of Medicine, Cukurova University \\ PO box 01330, Adana, Turkey \\ E-mail: nebiled@hotmail.com \\ Selvi Asker \\ Department of Chest Diseases, Faculty of Medicine, Van Yuzuncu Yil University \\ Van, Turkey \\ E-mail: selviasker@gmail.com \\ Asli Atasoy \\ Institute of Addiction and Forensic Science, Cukurova University \\ PO box 01330, Adana, Turkey \\ E-mail: asliatsy@gmail.com
}

\begin{abstract}
Development and validation of analytical method for the identification and quantification of any drug in blood is vital significance to study drug metabolism and, link clinical results. A 39-year-old woman who arrived in the emergency unit due to bleeding on her mouth twice. In first arriving the hospital, the patient regularly has ingested warfarin for 12 years and has had never an event related-INR until now. There were radiological lesions of the patient, and the INR value was 12. After the treatment, it was suggested that the patient discontinue warfarin, and she was discharged. After 1.5 month, the patient developed severe hypoxia and the INR value was 9 in second visiting the hospital when she said that she didn't ingest the drug for ten days. After the patient entered dialysis for three days and isolated from outside, the INR and the other values was normal. A blood specimen was sent to our forensic toxicology laboratory with the suspicion of suicidal or homicidal warfarin poisoning. Warfarin concentration determined using validated method by liquid chromatography-tandem mass spectrometer was 2.16 $\mu \mathrm{g} / \mathrm{ml}$. Clinicians should be aware the importance of analytical toxicological analyses to assess a series of forensic and clinical toxicological investigations in analysis of post-mortem and ante-mortem blood samples.
\end{abstract}

Keywords: toxicology; analysis; warfarin; clinical; suicide; homicide.

\section{Special Issue of Health Sciences}

DOI: $10.7176 / J S T R / 6-03-04$

\section{Introduction}

Warfarin that is the vitamin $\mathrm{K}$ antagonist (VKA) is extensively used to prevent the prophylaxis and to treat thromboembolic disease such as atrial fibrillation, myocardial infarction, antiphospholipid syndrome and heart valve replacement as oral anticoagulation agents [1]. It was initially used as a pesticide against harmful rodents and then it has been used widely as a therapeutic drug for the early 
1950s. Acute anticoagulant intoxication is rare, and it also has been the subject of various forensic cases [2-4]. Perhaps the most famous of these cases is the allegation that the Soviet leader Stalin was poisoned with warfarin in 1953 [5]. Most of overdose warfarin intake is associated with the patient who are prescribed anticoagulation drug to prevent mechanical valve prophylaxis or thromboembolism [6].

Warfarin that has high bioavailability is rapidly and completely absorbed from the gastrointestinal tract, and increases maximal blood levels in healthy volunteers in $90 \mathrm{~min}$ after oral administration. It is highly protein bound ( $>98 \%$ ), primarily to albumin. Only the free drug is pharmacologically active [7]. It has a half-life of 36 to $42 \mathrm{~h}$, and accumulates in the liver where it is predominantly metabolized by CYP-2CP microsomal liver enzymes. Its metabolism is affected by several factors such as diet, various herbal ingestion, environmental factors, interaction with other drugs [8] and, genetic polymorphism, particularly CYP2 mutations complicated which can alter warfarin metabolism leading to poisoning $[1,9,10]$. Warfarin has relatively narrow therapeutic window so hemorrhage complicate this treatment [9]. Overanticoagulation is encountered commonly. Such cases could be accidental, homicidal or suicidal intake of warfarin [11].

Warfarin toxicity clinically can be diagnosed by the Prothrombin Time (PT) test, showing the functions of the extrinsic coagulation system. Since the variability of the test tubes, measuring devices and measurement materials used influenced the test results, it was decided to express the PT test with a standard value (INR = International Normalized Ratio) in patients who used warfarin. Normal level of INR is around 1, what is this value in people using warfarin is determined by physicians. The targeted INR value is usually 2 to 3.5 in patients with deep vein thrombosis/pulmonary embolism, with brainheart vessel obstruction cardiac arrhythmias such as atrial fibrillation or heart valve disease [12]. But these tests might be influenced by several other diseases [11]. Several analytical methods and techniques have been used in order to detect and quantify anticoagulant substance in biological samples including autopsy materials for predicting the duration of vitamin K1 therapy or evaluating intoxication until recent $[13,14]$.

The objective of this study was to report a case of warfarin intake with diffuse alveolar hemorrhage evaluating by developing and validating highly sensitive and specific new analytical method to quantify warfarin level in blood rather than common clinical diagnosis whose hemostatic parameters might be manipulated by several other diseases other than anticoagulant intake in order to demonstrate drug intake and link clinical results for forensic and clinical purposes.

\section{Materials and Methods}

\subsection{Case history}

A 39-year-old woman (weighting approximately $65 \mathrm{~kg}$ ) who arrived in the emergency unit (Faculty of Medicine Hospital, Van Yüzüncü Yıl University, Van, Turkey) alone due to bleeding from her mouth at March 2018. The patient who underwent a mitral valve replacement 12 years ago regularly has ingested warfarin and has had never an event related-INR for 12 years. As she visited in emergency unit, the first INR value monitoring was 12 and the $\mathrm{Hb}$ (hemoglobin) value was $9 \mathrm{~g} / \mathrm{dl}$. There were extensive rhonchi and radiological lesions of the patient with alveolar haemorrhage (Figure 2). The hemorrhage associated with the patient's high INR value. The serum level of IgE was high. The INR value returned normal level after the fresh-freeze plasma (FFP) and low dose steroid were given to the patient so the patient was discharged. She consulted secondly to the emergency unit due to haemoptysis after 1.5 months and the INR value was 17 . Bilateral diffuse alveolar infiltration areas and patchy ground-glass opacity were observed by Chest X-ray and computed tomography throughout both lungs of the patient (Figure 3). She said that she has not ingested warfarin or any other drug and any herb other than $4 \mathrm{mg}$ steroid for ten days. The FFP were given to the patient again and the INR decreased 2 after two days. But haemoptysis increased after 3 days and INR increased 4 . Then the patient developed severe hypoxia and the INR increased 9. After the patient entered dialysis for 3 days and isolated from patient's relatives, the INR was normal level and the other clinical conditions were stable so she was discharged from the hospital. She has married and has not children until now, hence her husband has married a second wife. Her husband has not come to visit the patient during hospitalization and the patient has lived with her husband and her co-wife together. Because of this special situation and the healing after entering the patient into dialysis, the physicians in chest diseases unit of the hospital suspected that she could take warfarin or another rodenticide and obtained peripheral blood sample from the patient for toxicological analysis on 7 days after second hospitalization.

\subsection{Toxicological Analysis}

Peripheral blood specimen collected from the patient was sent to Forensic Toxicology Laboratory in the Department of Forensic Medicine, Çukurova University, Adana, Turkey because there have been no

20 | P a g e

www.iiste.org 
scientist and equipment to perform analytically warfarin level in blood in nearby regions. $1 \mathrm{ml}$ of blood sample was obtained. Internal standard (10 ng diazepam-d5) was added to $1 \mathrm{~mL}$ blood sample. Then, it was reconstituted in $2 \mathrm{~mL}$ of phosphate buffered saline (PBS). Then it was centrifuged for $10 \mathrm{~min}$ at 3500 rpm. Warfarin was extracted from blood using solid phase extraction columns (Oasis ${ }^{\circledR}$ HLB cartridge column; $3 \mathrm{cc}, 60 \mathrm{mg}$ ). Cartridges were conditioned respectively with $2 \mathrm{~mL}$ methanol, $2 \mathrm{~mL}$ distilled water and $2 \mathrm{~mL}$ PBS (all at $1 \mathrm{~mL} / \mathrm{min}$ ). Sample whose total volume was $3 \mathrm{~mL}$ were loaded on the SPE cartridge

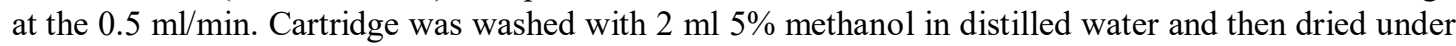
vacuum at approximately $125 \mathrm{mgHg}$ for $5 \mathrm{~min}$. Sample was eluted $2 \mathrm{ml}$ acetone:methanol (70:30 v/v) and $2 \mathrm{ml} 2 \% \mathrm{NH} 4 \mathrm{OH}$ in ethyl acetate $(98: 2 \mathrm{v} / \mathrm{v})$. Then extract was evaporated under nitrogen gas. Dry extracted blood sample was reconstituted in $1 \mathrm{ml}$ methanol, diluted 1:25 and 10 $\mu \mathrm{L}$ of it was injected onto the LC/MS/MS system. LC/MS/MS analysis was performed on a Shimadzu CBM-20A Ultra Flow Liquid Chromatography UFLC equipped with a Shimadzu SIL-20A/HT autosampler system and Shimadzu 8040 LC/MS/MS Systems. The separation of analyte was carried out using an pentafluorophenylpropyl (PFPP) column (Allure 50x2.150 mm i.d., $5 \mu \mathrm{m}$, Restek, Bellefonte, PA, USA), maintained at $40{ }^{\circ} \mathrm{C}$. LC/MSMS was operated in the Multiple Reaction Monitoring (MRM). The total analysis lasted 20 min.

Table 1. Operating conditions for LC/MS/MS analysis

\begin{tabular}{ll}
\hline Column & Restek PFPP Allure 50x2.150 mm i.d., $5 \mu \mathrm{m}$ \\
Injection volume & $10 \mu \mathrm{L}$ \\
Oven temperature & $40{ }^{\circ} \mathrm{C}$ \\
Mobile phase A & $10 \mathrm{mM}$ ammoniumacetate in Water \\
Mobile phase B & Methanol (LC grade) \\
Flow & $0.4 \mathrm{~mL} \cdot \min ^{-1}$ \\
Gradient & $0-0.01 \mathrm{~min} 5 \%$ solvent B \\
& $0-10$ min linear gradient to $95 \%$ solvent B \\
& $10-15$ min $95 \%$ solvent B \\
& $15-15.01$ min gradient to $5 \%$ solvent B \\
Detector & 20 min stop \\
Interface & Triple quadrupole mass spectrometer \\
Interface voltage & Electrospray ionisation \\
Nebulizing gas flow & $4.5 \mathrm{kV}$ \\
\hline
\end{tabular}

Table 2. The analytical LC-MS/MS parameters for Warfarin and Diazepam-d5(IS).

\begin{tabular}{lllll} 
& Parent Ion (m/z) & Product ions (m/z) & CE (Voltage) & RT (min) \\
\cline { 1 - 4 } Warfarin & \multirow{2}{*}{309.15} & 163.05 & -16 & \multirow{2}{*}{6.26} \\
\cline { 3 - 5 } Diazepam-d5 (IS) & \multirow{2}{*}{290.00} & 251.00 & -20 & \multirow{2}{*}{8.46} \\
\cline { 3 - 5 } & & 198.00 & -34 & \\
\hline
\end{tabular}

CE: collision energy, RT: retention time

Warfarin reference standard and diazepam-d5 internal standard were respectively purchased from United States Pharmacopeia (USP) and from RESTEK (Bellefonte, PA). Method validation was partly done for accuracy, selectivity, linearity, limit of detection (LOD), lowest limit of quantitation (LLOQ), dilution integrity, precision, carry-over, matrix effect according to the Scientific Working Group for Forensic Toxicology (SWGTOX) Standard Practices [15]. For development and validation of LC-MS/MS method for analysing warfarin in whole blood, calibrators and controls at certain concentrations were prepared by using blank blood samples.

\section{Results and discussion}

Firstly, the blood sample was found positive for warfarin by LC/MS-MS clinical toxicology screening method and then it was performed quantitation of warfarin in blood sample of the patient by LC/MSMS. The retention time was 6.4 min for warfarin and $8.5 \mathrm{~min}$ for diazepam-d5 (internal standard). According to method validation results, the intra-assay and inter-assay coefficient of variation was less than $8.6 \%$ and the absolute recovery value was $94 \%$ at $25 \mathrm{ng} / \mathrm{ml}$ concentration. The calibrator range

21 | P a g e 
established for warfarin were 1-100 $\mathrm{ng} / \mathrm{ml}$. The calibration curves observed good linearity with correlation coefficients greater than 0.999 . To assess dilution integrity, 1:25 dilution ratio of warfarin in whole blood was used and its bias range was 4.6 to $8.4 \%$. No marked matrix effect and carryover for warfarin was observed in the quality control samples at all tested concentrations. Limit of detection (LOD) and lowest limit of quantitation (LOQ) respectively were $0.08 \mathrm{ng} / \mathrm{ml}$ and $0.23 \mathrm{ng} / \mathrm{ml}$.

Warfarin concentration in the blood sample obtained on 7 days of after patient's second hospitalization when the patient said she had not ingested any drug for ten days, which quantified using LC/MS-MS with MRM method was $2.16 \mu \mathrm{g} / \mathrm{ml}$ that was calculated by taking into account the dilution factor $(1: 25)$ (fig. 1).
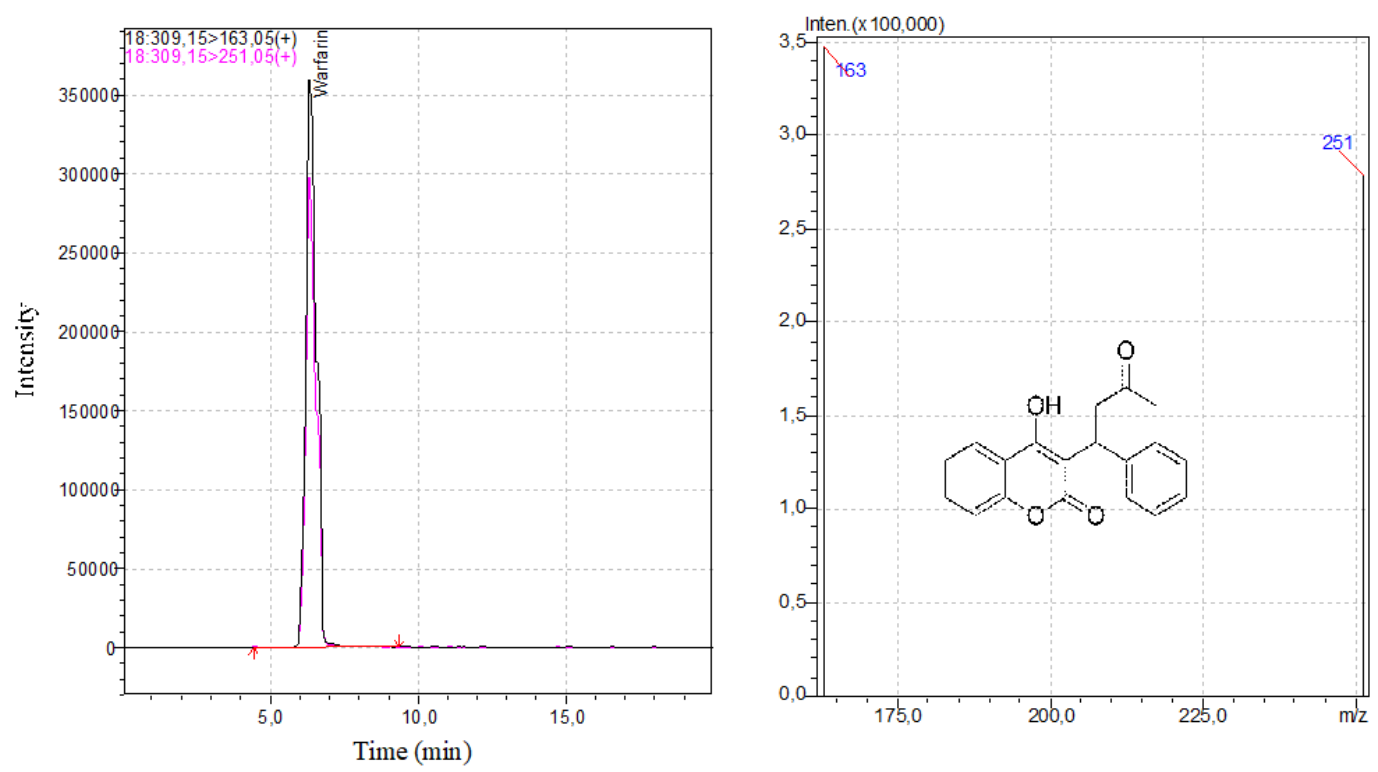

Figure 1. Ion chromatogram and mass spectrum of the blood sample containing warfarin $(86.2 \mathrm{ng} / \mathrm{ml}$, diluted with 1:25), (+) ESI.

The INR values was high until entering dialysis and the other treatments. The INR values of the patient who was developed severe hypoxia and whose the INR value increased 9 was normal level and the other clinical conditions after the patient entered dialysis for 3 days and isolated from patient's relatives. Chest $\mathrm{X}$ ray and high resolution $\mathrm{CT}$ of the chest scan showed signs of bilateral diffuse alveolar infiltration areas and patchy ground glass opacity at patient's both hospitalizations (fig. 2 and fig 3.).

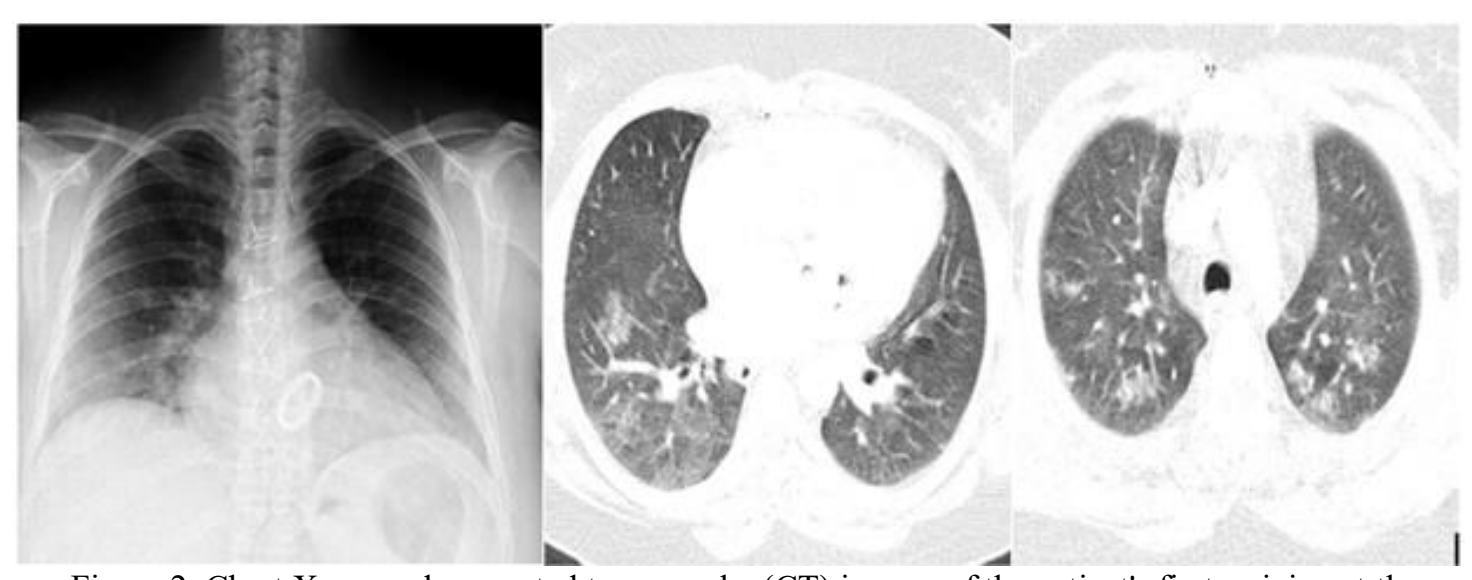

Figure 2. Chest X-ray and computed tomography (CT) images of the patient's first arriving at the hospital. In both lungs, diffuse nonspecific alveolar infiltration areas and patchy ground-glass opacity were observed. 


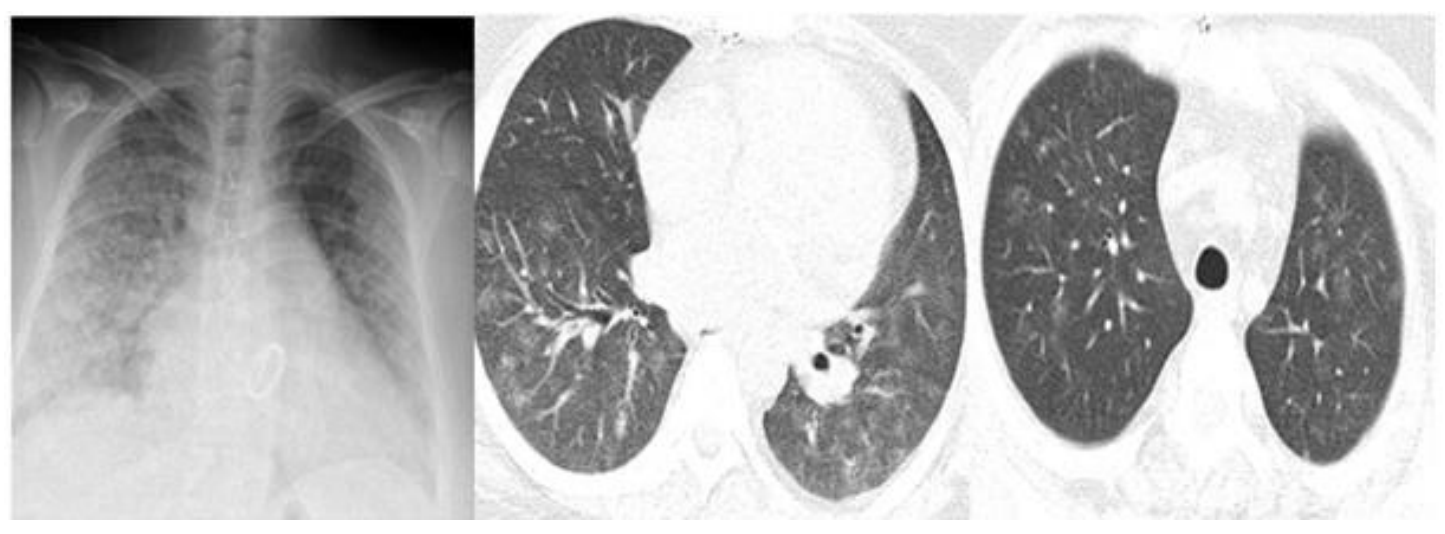

Figure 3. Chest X-ray and computed tomography (CT) images of the patient's second arriving at the hospital. Diffuse nonspecific alveolar infiltration areas and bilateral patchy ground glass opacity were observed in a patient with diffuse alveolar.

Warfarin which was originally used as rodenticide for many years in order to fight against rodents in 1948 and has been approved as pharmaceutic drug until now is a rare drug of choice for attempted homicide and suicide [16]. Its mechanism of action depends on inhibition of vitamin K epoxide reductase enzyme which results in depletion of the reduced form of vitamin K. Vitamin K is cofactor for the posttranslational carboxylation of glutamate residues to gamma-carboxyglutamates on the $\mathrm{N}$-terminal areas of vitamin K-dependent proteins. There are coagulation factors (factors II, VII, IX, and X) requiring gamma-carboxylation for biological activity. Warfarin occurs its anticoagulation effect by inhibition of vitamin $\mathrm{K}$ conversion cycle, thereby causing hepatic production of partially carboxylated and decarboxylated proteins with reduced procoagulation activity [1]. Diffuse alveolar haemorrhage (DAH) that could be caused by reducing coagulation activity is a catastrophic clinical syndrome which result from widespread bleeding in the acinar portion of the lung. DAH is an uncommon complication which is a small proportion ş.ely with overdose warfarin taken by children and adults for suicide [17,18].

In our case, it was developed diffuse alveolar hemorrhage with patchy ground glass opacity induced by warfarin intake for suicide or homicide according to radiological and toxicological findings. The suspicion that she could take warfarin or another rodenticide was occurred after the patient entered dialysis for 3 days and isolated from patient's relatives while the patient developed severe hypoxia and the INR raised to 9. The physicians suspected that the patient could take warfarin herself as a suicide attempt or it could be given her from the outside as a homicidal attempt. The forensic or clinical laboratories use often from the appearance of liquid chromatography-tandem mass spectrometry (LC$\mathrm{MS} / \mathrm{MS}$ ) for detection of warfarin or the other anticoagulant drugs in body fluid such as blood and, urine. Hence, toxicological analysis was performed in the patient's peripheral blood sample that obtained on patient's second admission to the hospital when the patient said she had not ingested for ten days using validated LC-MS/MS method by us and, warfarin concentration was determined $2.16 \mu \mathrm{g} / \mathrm{ml}$. This level was not exceeding the therapeutic concentration (1.0 to $3.1 \mu \mathrm{g} / \mathrm{mL})$ [19]. But if taking account into hospitalization and dialysis time and, the biological half-life of warfarin, it can ambiguously be said that the case is acute or chronic overdose warfarin poisoning because blood sampling time was seven days after the second hospitalization and, patient's amount and time of the drug ingestion were not known by us. Nevertheless, that warfarin was measured high concentration $(2.16 \mu \mathrm{g} / \mathrm{ml})$ in blood of the patient in 7 days after second visiting the hospital when she said that she didn't ingest the drug for ten have been considered as forensic evidence.

Previous studies in the literature have reported cases and analytical methods related with warfarin overdose intake. Yan $\mathrm{H}$ et al. analysed the incidence of 117 cases of poisoning caused by 13 anticoagulant drugs, including warfarin, in Eastern China [11]. Previous LC-MS-MS technique with liquid-liquid extraction method was used for the analysis of these drugs in blood samples. Limit of detection was 5 $\mathrm{ng} / \mathrm{ml}[20]$. Warfarin was found in blood samples of two patients $(1.7 \%)$ with concentrations $(0.4$ and 2.4 $\mu \mathrm{g} / \mathrm{mL})[11]$. Erdogan et al. reported a case with similar clinical outcomes compared to our case. Bilateral diffuse alveolar haemorrhage chest radiography was observed in their case that was not done analytic assessment [17]. In the post-mortem study, Gilard-Pioc et al. reported a case of sudden death with spontaneous epiglottic hematoma that warfarin in blood specimens collected from autopsy were 
measured at concentration in peripheral blood of 8.4 and $9.5 \mu \mathrm{g} / \mathrm{mL}$ in cardiac blood sample. But they clearly did not report which analytical devices and methods they used for quantitative analysis of warfarin and detail of the analytical method. In the autopsy findings, epiglottic hematoma spreading into surrounding soft tissues, completely obstructing the upper airway and blood in urine as hemorrhagic complication with associate warfarin intake were observed [2]. Levine et al. reported a total of 23 warfarin overdose cases between January 1987 and July 2012 in the retrospective study that analytical evaluation was not performed, and documented to be suicide attempt in 21 patients [6]. Bidny et al. developed and validated an LC-MS-MS method to quantitate nine rodenticides including warfarin in blood. The extraction method based on liquid-liquid extraction and LOQ for warfarin was $2 \mathrm{ng} / \mathrm{ml}$ [13]. In other case report related with warfarin poisoning, Berling et al. followed the concentrations of warfarin in blood using validated LC-MS-MS method after precipitating proteins with acetonitrile, and INR values during treatment. The warfarin level was $74.6 \mu \mathrm{g} / \mathrm{ml}$ at $26 \mathrm{~h}$ after ingestion and decreased to $3.7 \mu \mathrm{g} / \mathrm{ml}$ over $72 \mathrm{~h}$. It was observed that the INR value decreased with decreasing the concentration of warfarin [21]. In our case, unfortunately, decreasing or increasing the concentration of warfarin was not followed due to long distance between the hospital and the forensic toxicology laboratory where the blood specimen was analysed for transferring the specimen and insufficient biological sampling from the patient.

Toxicological analyses are of great benefit to investigate for predicting the duration of vitamin K1 therapy or evaluating intoxication. We confirmed analytically clinical toxicity caused by warfarin intake from our findings in order to determine whether the patient ingested warfarin at second visiting the hospital when she said that she didn't ingest the drug for ten days using LC-MS-MS method for the forensic investigation. But the overdose warfarin case cannot be demonstrated definitively whether an overdose of warfarin is an outcome of a suicide or homicide by us. More juridical investigations are necessary to determine the situation.

\section{Conclusions}

Specific and sensitive analytical methods such as LC-MS-MS method in quantitative analysis of warfarin have been required because INR test and the other common clinical diagnostic tests whose haemostatic parameters might be manipulated by several other diseases other than anticoagulant intake. A validated highly specific and sensitive LC-MS-MS method can be approved to be effective to detect the level of warfarin in blood for forensic and clinical purpose. Development and validation analytical method for the identification and quantification of warfarin in blood is vital significance to study drug metabolism, and link clinical results.

Our method was validated according to international guideline and can be successfully used to a series of forensic and clinical toxicological investigations in analysis of post-mortem and ante-mortem blood samples.

\section{Conflict of interest statement}

We declare that there isn't conflict of interest for the article: "Toxicological analysis of warfarin in an adult developing diffuse alveolar hemorrhage: a case report and literature review" and that this study did not receive any specific grant from funding agencies in the public, commercial, or not-for-profit sectors.

\section{Footnote}

This study was presented a poster presentation at 3rd Regional The Association of Forensic Toxicologists (TIAFT) Meeting in Turkey (October 18-20, 2018, in Nevsehir, Turkey)

\section{References}

[1] Hirsh J, Dalen JE, D.R A, et al (2001) Oral Anticoagulants: Mechanism of Action, Clinical Effectiveness, and Optimal Therapeutic Range. Chest 119:8S-21S. https://doi.org $/ 10.1378 /$ chest. 119.1

[2] Gilard-Pioc S, Guerard P, Paraf F, François-Purssell I (2017) Sudden Death by Spontaneous Epiglottic Hematoma Secondary to High Blood Levels of Warfarin. J Forensic Sci 62:10941096. https://doi.org/10.1111/1556-4029.13384

[3] Gill JR, Goldfeder LB, Hirsch CS (2006) Use of "therapeutic complication" as a manner of death. J Forensic Sci 51:1127-1133. https://doi.org/10.1111/j.1556-4029.2006.00222.x 
[4] Hosseini M, Hosseinzadeh A, Raufian K, Hedjazi A (2015) Nontraumatic retroperitoneal hematoma after warfarin administration: Fatal case report and review of the literature. Am J Forensic Med Pathol 36:245-248. https://doi.org/10.1097/PAF.0000000000000190

[5] Faria MA(2011) Stalin's mysterious death. Surg Neurol Int 2:161. https://doi.org/10.4103/21527806.89876

[6] Levine M, Pizon AF, Padilla-Jones A, Ruha AM (2014) Warfarin Overdose: A 25-Year Experience. J Med Toxicol 10:156-164. https://doi.org/10.1007/s13181-013-0378-8

[7] Jaffer A, Bragg L (2003) Practical tips for warfarin. Cleve Clin J Med 70:361-371. https://doi.org/10.3949/ccjm.70.4.361

[8] Launiainen T, Sajantila A, Rasanen I, et al (2010) Adverse interaction of warfarin and paracetamol: Evidence from a post-mortem study. Eur J Clin Pharmacol 66:97-103. https://doi.org/10.1007/s00228-009-0727-3

[9] Mendonca S, Gupta D, Valsan A, Tewari R (2017) Warfarin related acute kidney injury: A case report. Indian J Nephrol 27:78-80. https://doi.org/DOI: 10.4103/0971-4065.177142

[10] Piatkov I, Rochester C, Jones T, Boyages S (2010) Warfarin Toxicity and Individual VariabilityClinical Case. Toxins (Basel) 2:2584-2592. https://doi.org/10.3390/toxins2112584

[11] Yan H, Zhu L, Zhuo X, et al (2016) Anticoagulant rodenticide intoxication in east China: a threeyear analysis. Forensic Sci Res 1:22-27. https://doi.org/10.1080/20961790.2016.1242042

[12] Wittkowsky AK, Devine EB (2004) Frequency and causes of overanticoagulation and underanticoagulation in patients treated with warfarin. Pharmacotherapy 24:1311-1316. https://doi.org/10.1592/phco.24.14.1311.43144

[13] Bidny S, Gago K, David M, et al (2015) A validated LC-MS-MS method for simultaneous identification and quantitation of rodenticides in blood. J Anal Toxicol 39:219-224. https://doi.org/10.1093/jat/bku175

[14] Dias E, Hachey B, McNaughton C, et al (2013) An LC-MS assay for the screening of cardiovascular medications in human samples. J Chromatogr B Anal Technol Biomed Life Sci 937:44-53. https://doi.org/10.1016/j.jchromb.2013.08.010

[15] Scientific Working Group for Forensic Toxicology (2013) Scientific Working Group for Forensic Toxicology (SWGTOX) standard practices for method validation in forensic toxicology. J Anal Toxicol 37:452-474. https://doi.org/10.1093/jat/bkt054

[16] Holmes RW, Love J (1952) Suicide attempt with warfarin, a bishydroxycoumarin-like rodenticide. J Am Med Assoc 148:935-937. https://doi.org/10.1001/jama. 1952.62930110003013a

[17] Erdogan D, Kocaman O, Oflaz H, Goren T (2004) Alveolar hemorrhage associated with warfarin therapy: A case report and literature review. Int J Cardiovasc Imaging 20:155-159. https://doi.org/10.1023/B:CAIM.0000014101.06445.66

[18] Heffler E, Campisi R, Ferri S, Crimi N (2016) A Bloody Mess: An Unusual Case of Diffuse Alveolar Hemorrhage because of Warfarin Overdose. Am J Ther 23:e1280-e1283. https://doi.org/10.1097/MJT.0000000000000397

[19] Winek CL, Wahba WW, Winek Jr. CL, Winek Balzer T (2001) Drug and chemical blood-level data 2001. Forensic Sci Int 122:107-123 
[20] Fourel I, Hugnet C, Goy-Thollot I, Berny P (2010) Validation of a new liquid chromatographytandem mass spectrometry ion-trap technique for the simultaneous determination of thirteen anticoagulant rodenticides, drugs, or natural products. J Anal Toxicol 34:95-102. https://doi.org/10.1093/jat/34.2.95

[21] Berling I, Mostafa A, Grice JE, et al (2017) Warfarin Poisoning with Delayed Rebound Toxicity. J Emerg Med 52:194-196. https://doi.org/10.1016/j.jemermed.2016.05.068 